Georgian Mathematical Journal

Volume 11 (2004), Number 3, 495-514

\title{
EFFECTIVE SOLUTION OF A CLASS OF BOUNDARY VALUE PROBLEMS OF THERMOELASTICITY IN GENERALIZED CYLINDRICAL COORDINATES
}

\author{
N. KHOMASURIDZE
}

\begin{abstract}
A class of static boundary value problems of thermoelasticity is effectively solved for bodies bounded by coordinate surfaces of generalized cylindrical coordinates $\rho, \alpha, z$ ( $\rho, \alpha$ are orthogonal curvilinear coordinates on the plane and $z$ is a linear coordinate). Besides in the Cartesian system of coordinates some boundary value thermoelasticity problems are separately considered for a rectangular parallelepiped. An elastic body occupying the domain $\Omega=\left\{\rho_{0}<\rho<\rho_{1}, \alpha_{0}<\alpha<\alpha_{1}, 0<z<z_{1}\right\}$, is considered to be weakly transversally isotropic (the medium is weakly transversally isotropic if its nine elastic and thermal characteristics are correlated by one or several conditions) and non-homogeneous with respect to $z$.
\end{abstract}

2000 Mathematics Subject Classification: 74B05, 74F05.

Key words and phrases: Thermoelasticity, symmetry condition, curvilinear coordinates, Laplace field, Fourier method.

\section{INTRODUCTION}

The given work, precise solutions for a class of static boundary value thermoelasticity problems for a weakly transversally isotropic $(z=$ const is the plane of isotropy) a section of a finite, annular cylinder $\Omega=\left\{\rho_{0}<\rho<\rho_{1}\right.$, $\left.\alpha_{0}<\alpha<\alpha_{1}, 0<z<z_{1}\right\}$ non-homogeneous with respect to $z$ in generalized cylindrical coordinates $\rho, \alpha$, $z$, with Lame coefficients $h_{\rho}=h_{\alpha}=h(\rho, \alpha), h_{z}=1$ [1]. The elastic medium is called weakly transversally isotropic when its nine elastic and thermal characteristics are correlated by certain conditions. These conditions are not artificial since they are automatically satisfied in the case of homogeneous isotropic media. The first part of the paper is focused on thermoelasticity problems when on the four lateral cylindrical surfaces of a section of a finite, annular cylinder (SFAC) $\rho=\rho_{0}, \rho=\rho_{1}, \alpha=0$ and $\alpha=\alpha_{1}$ homogeneous boundary symmetry- or antisymmetry-type conditions are defined [2] while on $z=z_{j}\left(j=0,1 ; z_{0}=0\right)$ we have: arbitrary thermal disturbance and equal to zero normal displacement and tangential stresses or normal stress and tangential displacements. Besides, the thermoelastic equilibrium of an SFAC multilayer along $z$ is considered. The second part of the article is devoted to the thermoelastic equilibrium of a rectangular parallelepiped when on each of its six sides the following values are defined: the temperature and equal to zero normal stress and tangential displacements or the normal derivative of the temperature 
(thermal flow density) and equal to zero normal displacement and tangential stresses.

The obtained solutions are considerable practical importance. In particular, they can be used to determine thermoelastic stresses in thick construction plates of various shape; in bodies of complex shape affected by smooth or rough rigid dies of various shape; in construction slabs with easily deformable aggregate, etc. The solution of boundary value problems involving SFACs only affected by thermal fields can be used in metallurgy.

In the Appendix a specific problem is solved.

\section{Equilibrium Equations, Boundary Conditions, General SOLUTION}

The thermoelastic equilibrium of a transversally isotropic body when mass forces are absent can be described in generalized cylindrical coordinates $\rho, \alpha, z$ in the following way [3]:

$$
\begin{aligned}
& \frac{\partial}{\partial \rho}\left(h R_{\rho}\right)+\frac{1}{h} \frac{\partial}{\partial \alpha}\left(h^{2} R_{\alpha}\right)+h^{2} \frac{\partial R_{z}}{\partial z}-\frac{\partial h}{\partial \rho} A_{\alpha}=0 \\
& \frac{\partial}{\partial \alpha}\left(h A_{\alpha}\right)+h^{2} \frac{\partial A_{z}}{\partial z}+\frac{1}{h} \frac{\partial}{\partial \rho}\left(h^{2} A_{\rho}\right)-\frac{\partial h}{\partial \alpha} R_{\rho}=0 \\
& h^{2} \frac{\partial Z_{z}}{\partial z}+\frac{\partial}{\partial \rho}\left(h Z_{\rho}\right)+\frac{\partial}{\partial \alpha}\left(h Z_{\alpha}\right)=0 .
\end{aligned}
$$

$R_{\rho}, A_{\alpha}, Z_{z}$ are normal stresses, $R_{\alpha}=A_{\rho}, R_{z}=Z_{\rho}, A_{z}=Z_{\alpha}$ are tangential stresses and the equalities correlating the stresses and the displacements have the following form:

$$
\begin{aligned}
R_{\rho}= & c_{1} e_{\rho \rho}+\left(c_{1}-2 c_{5}\right) e_{\alpha \alpha}+c_{3} e_{z z}-k_{10} T=\frac{c_{1}}{h^{2}}\left(\frac{\partial(h u)}{\partial \rho}+\frac{\partial(h v)}{\partial \alpha}\right) \\
& -2 c_{5}\left(\frac{1}{h} \frac{\partial v}{\partial \alpha}+\frac{1}{h^{2}} \frac{\partial h}{\partial \rho} u\right)+c_{3} \frac{\partial w}{\partial z}-k_{10} T \\
A_{\alpha}= & c_{1} e_{\alpha \alpha}+\left(c_{1}-2 c_{5}\right) e_{\rho \rho}+c_{3} e_{z z}-k_{10} T=\frac{c_{1}}{h^{2}}\left(\frac{\partial(h u)}{\partial \rho}+\frac{\partial(h v)}{\partial \alpha}\right) \\
& -2 c_{5}\left(\frac{1}{h} \frac{\partial u}{\partial \rho}+\frac{1}{h^{2}} \frac{\partial h}{\partial \alpha} v\right)+c_{3} \frac{\partial w}{\partial z}-k_{10} T, \\
Z_{z}= & c_{2} e_{z z}+c_{3}\left(e_{\rho \rho}+e_{\alpha \alpha}\right)-k_{20} T=\frac{c_{3}}{h^{2}}\left(\frac{\partial(h u)}{\partial \rho}+\frac{\partial(h v)}{\partial \alpha}\right) \\
& +c_{2} \frac{\partial w}{\partial z}-k_{20} T, \\
Z_{\rho}= & c_{4} e_{z \rho}=c_{4}\left(\frac{\partial u}{\partial z}+\frac{1}{h} \frac{\partial w}{\partial \rho}\right), Z_{\alpha}=c_{4} e_{z \alpha}=c_{4}\left(\frac{1}{h} \frac{\partial w}{\partial \alpha}+\frac{\partial v}{\partial z}\right) \\
A_{\rho}= & c_{5} e_{\alpha \rho}=c_{5}\left[\frac{\partial}{\partial \rho}\left(\frac{v}{h}\right)+\frac{\partial}{\partial \alpha}\left(\frac{u}{h}\right)\right]
\end{aligned}
$$


where $u, v, w$ are the components of the displacement vector $\vec{U}$ along the tangents to the coordinate lines $\rho, \alpha, z ; e_{\rho \rho}, e_{\alpha \alpha}, e_{z z}, e_{z \rho}=e_{\rho z}, e_{z \alpha}=e_{\alpha z}, e_{\alpha \rho}=e_{\rho \alpha}$ are deformations; $c_{j}=c_{j}(z)(j=1,2, \ldots, 5)$ are elastic characteristics where [4] $c_{1}>0, c_{2}>0, c_{4}>0, c_{5}>0, c_{1} c_{2}>c_{3}^{2}+c_{2} c_{5}$ (free energy is a positively defined square form without the inequality $c_{2}>0$, although the condition $c_{2}>0$ is almost always satisfied); $k_{10}=\left[2\left(c_{1}-c_{5}\right) k_{1}+c_{3} k_{2}\right], k_{20}=\left(2 c_{3} k_{1}+c_{2} k_{2}\right)$, and $k_{1}=k_{1}(z) \quad k_{2}=k_{2}(z)$ are linear thermal expansion coefficients in the plane of isotropy $z=$ const and along $z, k_{1}>0, k_{2}>0 ; T$ is the change in the temperature of the elastic body defined by the equation

$$
\Delta_{2} T+\frac{1}{\lambda_{1}} \frac{\partial}{\partial z}\left(\lambda_{2} \frac{\partial T}{\partial z}\right)=0
$$

and the corresponding boundary conditions (the temperature of the body in its natural state is taken as zero) while $\lambda_{1}=\lambda_{1}(z)$ and $\lambda_{2}=\lambda_{2}(z)$ are the heat conduction coefficients in the plane of isotropy and along $z, \lambda_{1}>0, \lambda_{2}>0$; $\Delta_{2}=\frac{1}{h^{2}}\left(\frac{\partial^{2}}{\partial \rho^{2}}+\frac{\partial^{2}}{\partial \alpha^{2}}\right)$. Weak transversal isotropy conditions have the following form:
a) $c_{1} c_{2}=\left(c_{3}+2 c_{4}\right)^{2}$,
b) $k_{20}=c \cdot k_{10}$,
c) $c_{4}=$ const

where $c=\sqrt{\frac{c_{2}}{c_{1}}}$. Conditions (4) are not artificial since the automatically hold in the case of homogeneous isotropic media when $c_{1}=c_{2}=c_{3}+2 c_{4}, k_{10}=k_{20}$, $c=1$. Using (1) and (2) we can obtain the following system with respect to the functions $D, K_{\rho}, K_{\alpha}, K_{z}, u, v, w$ :
a) $\frac{\partial}{\partial z}(c \cdot D)-\frac{1}{h^{2}}\left[\frac{\partial\left(h K_{\alpha}\right)}{\partial \rho}-\frac{\partial\left(h K_{\rho}\right)}{\partial \alpha}\right]=0$
b) $\frac{\partial D}{\partial \rho}-\frac{\partial K_{z}}{\partial \alpha}+\frac{\partial\left(h K_{\alpha}\right)}{\partial z}=0$
c) $\frac{\partial D}{\partial \alpha}-\frac{\partial\left(h K_{\rho}\right)}{\partial z}+\frac{\partial K_{z}}{\partial \rho}=0$
d) $\frac{1}{h^{2}}\left[\frac{\partial\left(h K_{\rho}\right)}{\partial \rho}+\frac{\partial\left(h K_{\alpha}\right)}{\partial \alpha}\right]+\frac{\partial}{\partial z}\left(g K_{z}\right)=0$
e) $\frac{1}{h^{2}}\left[\frac{\partial(h u)}{\partial \rho}+\frac{\partial(h v)}{\partial \alpha}\right]+c \frac{\partial w}{\partial z}-\frac{k_{10}}{c_{1}} T=\frac{D}{c_{1}}$,
f) $\frac{\partial w}{\partial \alpha}-\frac{\partial(h v)}{\partial z}=\frac{h K_{\rho}}{c_{4}}$
g) $\frac{\partial(h u)}{\partial z}-\frac{\partial w}{\partial \rho}=\frac{h K_{\alpha}}{c_{4}}$,
h) $\frac{\partial(h v)}{\partial \rho}-\frac{\partial(h u)}{\partial \alpha}=\frac{h^{2} K_{z}}{c_{5}}$,

where $g=\frac{c_{4}}{c_{5}} ;(5 \mathrm{~d})$ is an identity, which immediately follows from $(5 \mathrm{f}, \mathrm{g}, \mathrm{h})$.

Further we shall consider the thermoelastic equilibrium of an SFAC occupying the domain $\Omega=\left\{\rho_{0}<\rho<\rho_{1}, \alpha_{0}<\alpha<\alpha_{1}, 0<z<z_{1}\right\}$. The boundary 
conditions that will appear have the following form:

For $\rho=\rho_{j}$ we have : a) $\frac{\partial T}{\partial \rho}=0, u=0, K_{z}=0, Z_{\rho}=0$ or

b) $T=0, \quad D=0, \quad v=0, \quad w=0$.

For $\alpha=\alpha_{j}$ we have : a) $\frac{\partial T}{\partial \alpha}=0, v=0, Z_{\alpha}=0, K_{z}=0$ or

b) $T=0, \quad D=0, \quad w=0, u=0$.

For $z=z_{j}$ we have : a) $T=\tau_{3 j}(\rho, \alpha)$ or b) $\frac{\partial T}{\partial z}=\widetilde{\tau}_{3 j}(\rho, \alpha)$, or

c) $\frac{\partial T}{\partial z}+\theta_{3 j} T=\widetilde{\tau}_{3 j}(\rho, \alpha)$

or $z=z_{j}$ we have :
a) $Z_{z}=F_{3 j 1}(\rho, \alpha), h Z_{\rho}=F_{3 j 2}(\rho, \alpha), h Z_{\alpha}=F_{3 j 3}(\rho, \alpha)$ or
b) $w=f_{3 j 1}(\rho, \alpha), \quad h u=f_{3 j 2}(\rho, \alpha), \quad h v=f_{3 j 3}(\rho, \alpha)$, or
c) $w=f_{3 j 1}(\rho, \alpha), h Z_{\rho}=F_{3 j 2}(\rho, \alpha), h Z_{\alpha}=F_{3 j 3}$, or
d) $Z_{z}=F_{3 j 1}(\rho, \alpha), h u=f_{3 j 2}(\rho, \alpha), h v=f_{3 j 3}(\rho, \alpha)$.

In (6)-(9) we have $j=0,1$, with $z_{0}=0 ; \theta_{3 j}$ are defined constants. The functions $\widetilde{\tau}_{3 j}(\rho, \alpha), F_{3 j 1}(\rho, \alpha)$ themselves, the functions $\tau_{3 j}(\rho, \alpha), f_{3 j 1}(\rho, \alpha), F_{3 j 2}(\rho, \alpha)$ and $F_{3 j 3}(\rho, \alpha)$ with their first derivatives and the functions $f_{3 j 2}(\rho, \alpha)$ and $f_{3 j 3}(\rho, \alpha)$ with their first and second derivatives are assumed to expand into absolutely and uniformly converging Fourier series with respect to the eigen-functions of the corresponding Sturm-Liouville problems. Besides, the functions are chosen so that compatibility conditions hold on the edges of the SFAC. The interpretation of boundary conditions (6), (7) and conditions (9c), (9d) for $f_{3 j i}(\rho, \alpha)=0$, $F_{3 j i}(\rho, \alpha)=0(i=1,2,3)$ is given in [1].

The present paper deals with the construction of a regular solution of some kind of boundary value problem of thermoelasticity, so that it is necessary to define the concept of regularity.

The solution of system (5) defined by the functions $T, u, v, w$ will be called regular if $T$ is twice and $u, v, w$ is three times continuously differentiable in the domain $\widetilde{\Omega}$, where $\widetilde{\Omega}$ is the domain $\Omega$ with the boundaries $\rho=\rho_{j}$ and $\alpha=\alpha_{j}$, and on the surface $z=z_{j} T$ with its first derivatives and $u, v$ and $w$ with their first and second derivatives can be represented by absolutely and uniformly converging Fourier series with respect to eigen-functions of the corresponding Sturm-Liouville problems. In addition, we assume that the equilibrium equations hold for $\rho=\rho_{j}$ and $\alpha=\alpha_{j}$.

Theorem 1. For the class of thermoelasticity boundary value problems under consideration the general solution of system (5) in a class of regular functions can be represented as

$$
h u=\frac{\partial \varphi_{33}}{\partial \rho}+\frac{\partial \varphi_{32}}{\partial \alpha}, \quad h v=\frac{\partial \varphi_{33}}{\partial \alpha}-\frac{\partial \varphi_{32}}{\partial \rho}, \quad w=\frac{\partial \varphi_{33}}{\partial z}+\frac{\partial\left(c \cdot \varphi_{31}\right)}{\partial z},
$$


where

$$
\begin{aligned}
& \Delta_{2} \varphi_{31}+\frac{\partial^{2}}{\partial z^{2}}\left(c \cdot \varphi_{31}\right)=0, \\
& \Delta_{2} \varphi_{32}+g \frac{\partial^{2} \varphi_{32}}{\partial z^{2}}=0, \\
& \Delta_{2} \varphi_{33}+c \frac{\partial^{2} \varphi_{33}}{\partial z^{2}}=\frac{c_{4}-\sqrt{c_{1} c_{2}}}{c_{1}} \frac{\partial^{2}\left(c \cdot \varphi_{31}\right)}{\partial z^{2}}+\frac{k_{10}}{c_{1}} T .
\end{aligned}
$$

Proof. It follows from $(5 \mathrm{a}, \mathrm{b}, \mathrm{c})$ that

$$
\Delta_{2} D+\frac{\partial^{2}}{\partial z^{2}}(c \cdot D)=0 .
$$

Let

$$
D=c_{4} \frac{\partial^{2}}{\partial z^{2}}\left(c \cdot \varphi_{31}\right)
$$

where $\Delta_{2} \varphi_{31}+\frac{\partial^{2}}{\partial z^{2}}\left(c \cdot \varphi_{31}\right)=0$. The possible loss of generality in the expression for $D$, caused by the presence of the second derivative in the right-hand side of equality (12), can be reestablished by an appropriate representation of the function $\varphi_{31}$ using the method of separation of variables (the technique is similar to that in $[2])$.

Taking (12) into account we can write equation $(5 a, b, c)$ as

$$
\begin{gathered}
\frac{\partial}{\partial \rho}\left[h K_{\alpha}+c_{4} \frac{\partial^{2}\left(c \cdot \varphi_{31}\right)}{\partial \rho \partial z}\right]-\frac{\partial}{\partial \alpha}\left[h K_{\rho}-c_{4} \frac{\partial^{2}\left(c \cdot \varphi_{31}\right)}{\partial \alpha \partial z}\right]=0 \\
\frac{\partial}{\partial z}\left[h K_{\alpha}+c_{4} \frac{\partial^{2}\left(c \cdot \varphi_{31}\right)}{\partial \rho \partial z}\right]-\frac{\partial K_{z}}{\partial \alpha}=0 \\
\frac{\partial K_{z}}{\partial \rho}-\frac{\partial}{\partial z}\left[h K_{\rho}-c_{4} \frac{\partial^{2}\left(c \cdot \varphi_{31}\right)}{\partial \rho \partial z}\right]=0 .
\end{gathered}
$$

The latter equalities imply that a function $c_{4} \frac{\partial \varphi_{32}}{\partial z}$ exists, such that

$$
\begin{aligned}
h K_{\rho} & =c_{4} \frac{\partial}{\partial z}\left[\frac{\partial \varphi_{32}}{\partial \rho}+\frac{\partial\left(c \cdot \varphi_{31}\right)}{\partial \alpha}\right], \\
h K_{\alpha} & =c_{4} \frac{\partial}{\partial z}\left[\frac{\partial \varphi_{32}}{\partial \alpha}-\frac{\partial\left(c \cdot \varphi_{31}\right)}{\partial \rho}\right], \quad K_{z}=c_{4} \frac{\partial^{2} \varphi_{32}}{\partial z^{2}} .
\end{aligned}
$$

Substituting the expressions for $K_{\rho}, K_{\alpha}$, and $K_{z}$ into (5d) we obtain

$$
\text { a) } \frac{\partial}{\partial z}\left(\Delta_{2} \varphi_{32}+g \frac{\partial^{2} \varphi_{32}}{\partial z^{2}}\right)=0 \quad \text { or } \quad \text { b) } \Delta_{2} \varphi_{32}+g \frac{\partial^{2} \varphi_{32}}{\partial z^{2}}=f(\rho, \alpha) \text {. }
$$

A particular solution of equation (13b) can be found in the form of the function $\varphi_{32}^{*}=\varphi_{32}^{*}(\rho, \alpha)$ of two variables (indeed, if a particular solution is sought in the form of $\varphi_{32}^{*}=\varphi_{32}^{*}(\rho, \alpha)$, it leads to the equation $\Delta_{2} \varphi_{32}^{*}=f(\rho, \alpha)$, which implies that $\varphi_{32}^{*}$ will be a function of the variables $\rho$ and $\alpha$ ), and if that is true, then in the expressions for $K_{\rho}, K_{\alpha}$ and $K_{z}$ no $\varphi_{32}^{*}$ will appear, so with no loss of generality we assume $f(\rho, \alpha)=0$. 
Equalities $(5 f, g, h)$, taking into account the expressions for $K_{\rho}, K_{\alpha}$ and $K_{z}$, can be written as

$$
\begin{aligned}
& \frac{\partial}{\partial \alpha}\left[w-\frac{\partial\left(c \cdot \varphi_{31}\right)}{\partial z}\right]-\frac{\partial}{\partial z}\left(h v+\frac{\partial \varphi_{32}}{\partial \rho}\right)=0, \\
& \frac{\partial}{\partial z}\left(h u-\frac{\partial \varphi_{32}}{\partial \alpha}\right)-\frac{\partial}{\partial \rho}\left[w-\frac{\partial\left(c \cdot \varphi_{31}\right)}{\partial z}\right]=0 \\
& \frac{\partial}{\partial \rho}\left(h v+\frac{\partial \varphi_{32}}{\partial \rho}\right)-\frac{\partial}{\partial \alpha}\left(h u-\frac{\partial \varphi_{32}}{\partial \alpha}\right)=0 .
\end{aligned}
$$

The latter equalities suggest that a function $\varphi_{33}$ exists such that

$$
h u=\frac{\partial \varphi_{33}}{\partial \rho}+\frac{\partial \varphi_{32}}{\partial \alpha}, \quad h v=\frac{\partial \varphi_{33}}{\partial \alpha}-\frac{\partial \varphi_{32}}{\partial \rho}, \quad w=\frac{\partial \varphi_{33}}{\partial z}+\frac{\partial\left(\varphi_{31}\right)}{\partial z} .
$$

Substituting (14) into (5e) we have

$$
\Delta_{2} \varphi_{33}+c \frac{\partial^{2} \varphi_{33}}{\partial z^{2}}=\frac{c_{4}-\sqrt{c_{1} c_{2}}}{c_{1}} \frac{\partial^{2}\left(c \cdot \varphi_{31}\right)}{\partial z^{2}}+\frac{k_{10}}{c_{1}} T .
$$

Thus both equations (11) and representations (10) have been obtained, hence Theorem 1 is proved.

If we transform boundary conditions (9) in the same manner as in [2], we can construct the solution of any of boundary value problems (5)-(9) as well as of the corresponding boundary value-contact problems for a multilayer cylindrical body (the construction of the solution is as effective as in [2]; in the case of a non-homogeneous medium the specific type of non-homogeneity should be defined). Without dwelling on it since in [2] a broader (in the sense of elastic and thermal properties of the medium) class of boundary value and boundary value-contact problems of thermoelasticity is considered (on the other hand, system (11) is simpler than the corresponding system in [2]), we shall consider a special class of thermoelastic boundary value problems. With this aim we shall once more (for convenience) write boundary conditions $(9 \mathrm{c})$ and $(9 \mathrm{~d})$ for $f_{3 j i}(\rho, \alpha)=0$ and $F_{3 j i}(\rho, \alpha)=0(i=1,2,3)$.

$$
\begin{gathered}
\text { For } z=z_{j} \text { we have : a) } w=0, h Z_{\rho}=0, h Z_{\alpha}=0, \text { or } \\
\text { b) } Z_{z}=0, h u=0, h v=0 .
\end{gathered}
$$

\section{Thermoelasic Field With Zero $\operatorname{rot} \vec{U}$ and $D$}

Consider a class of boundary value problems of thermoelasticity generated by boundary value problems (5), (6), (7), (8), (15).

We shall find the solution of boundary value problems (5), (6), (7), (8), (15) assuming that $\varphi_{31}=0$ and $\varphi_{32}=0$ in (10) and (11). Then (10) and (11) will, respectively, take the following form:

$$
h u=\frac{\partial \varphi_{33}}{\partial \rho}, \quad h v=\frac{\partial \varphi_{33}}{\partial \alpha}, \quad w=\frac{\partial \varphi_{33}}{\partial z} ;
$$




$$
\Delta_{2} \varphi_{33}+c \frac{\partial^{2} \varphi_{33}}{\partial z^{2}}=\frac{k_{10}}{c_{1}} T
$$

Using (2) and (16), for a normal stress $Z_{z}$ (with (4a), (4b) and (17) in mind) and tangential stresses $Z_{\rho}$ and $Z_{\alpha}$ we have

$$
Z_{z}=-2 c_{4} \Delta_{2} \varphi_{33}, \quad Z_{\rho}=\frac{2 c_{4}}{h} \frac{\partial^{2} \varphi_{33}}{\partial \rho \partial z}, \quad Z_{\alpha}=\frac{2 c_{4}}{h} \frac{\partial^{2} \varphi_{33}}{\partial \alpha \partial z} .
$$

It is important that on the lateral surfaces $\rho=\rho_{j}$ and $\alpha=\alpha_{j}$ of a generalized cylinder the homogeneous boundary conditions for $\varphi_{33}$ and $T$ are the same. Indeed, when conditions (6a) are satisfied we have $\frac{\partial T}{\partial \rho}=0, \frac{\partial \varphi_{33}}{\partial \rho}=0$, when conditions (6b) and (7b) are satisfied we have $T=0, \varphi_{33}=0$, and when conditions (7) are satisfied we have $\frac{\partial T}{\partial \alpha}=0, \frac{\partial \varphi_{33}}{\partial \alpha}=0$.

It can be easily seen that conditions $\varphi_{31}=0$ and $\varphi_{32}=0 \operatorname{imply} \operatorname{rot} \vec{U}=0$ and $D=0$ or

$$
D=0, \quad K_{\rho}=0, \quad K_{\alpha}=0, \quad K_{z}=0 .
$$

In the forthcoming we will show that such kind of thermal field corresponds to the class of boundary value problems (5), (6), (7), (8), (15).

Let $\varphi_{33}^{0}$ and $\varphi_{33}^{*}$, respectively, denote the general and a particular solution of equation (17). Consider boundary conditions for equation (17) on the plane boundaries of the SFAC $z=0$ and $z=z_{1}$ (on each of the surfaces $\rho=\rho_{j}$ and $\alpha=\alpha_{j}$ either the functions $\varphi_{33}^{0}$ and $\varphi_{33}^{*}$ or their normal derivatives are equal to zero):

$$
\begin{aligned}
& \text { a) }\left.\varphi_{33}^{0}\right|_{z=0}+\left.\varphi_{33}^{*}\right|_{z=0}=0,\left.\quad \varphi_{33}^{0}\right|_{z=z_{1}}+\left.\varphi_{33}^{*}\right|_{z=z_{1}}=0 \\
& \text { b) }\left.\frac{\partial \varphi_{33}^{0}}{\partial z}\right|_{z=0}+\left.\frac{\partial \varphi_{33}^{*}}{\partial z}\right|_{z=0}=0,\left.\quad \frac{\partial \varphi_{33}^{0}}{\partial z}\right|_{z=z_{1}}+\left.\frac{\partial \varphi_{33}^{*}}{\partial z}\right|_{z=z_{1}}=0 \\
& \text { c) }\left.\varphi_{33}^{0}\right|_{z=0}+\left.\varphi_{33}^{*}\right|_{z=0}=0,\left.\quad \frac{\partial \varphi_{33}^{0}}{\partial z}\right|_{z=z_{1}}+\left.\frac{\partial \varphi_{33}^{*}}{\partial z}\right|_{z=z_{1}}=0 .
\end{aligned}
$$

In case of $(19 \mathrm{c})$ we consider $\left.\varphi_{33}^{*}\right|_{z=0}$ and $\left.\frac{\partial \varphi_{33}^{*}}{\partial z}\right|_{z=z_{1}}$ to be defined functions (the function $T$ in (17) is assumed to be known, resulting from the solution of a corresponding boundary value problem for equation (3)) and $\left.\varphi_{33}^{0}\right|_{z=0}$ and $\left.\frac{\partial \varphi_{33}^{0}}{\partial z}\right|_{z=z_{1}}$ are chosen so that conditions (19c) are satisfied. We behave in a similar way in the case of (19a) and (19b).

Conditions (19a) and formulas (16) and (18) imply

$$
\left.u\right|_{z=z_{j}}=0,\left.\quad v\right|_{z=z_{j}}=0,\left.\quad Z_{z}\right|_{z=z_{j}}=0 .
$$

Conditions (19b) and formulas (16) and (18) imply

$$
\left.Z_{\rho}\right|_{z=z_{j}}=0,\left.\quad Z_{\alpha}\right|_{z=z_{j}}=0,\left.\quad w\right|_{z=z_{j}}=0 .
$$

Conditions (19c) and formulas (16) and (18) imply

$$
u=0, \quad v=0, \quad Z_{z}=0 \text { for } z=0
$$


and

$$
Z_{\rho}=0, \quad Z_{\alpha}=0, \quad w=0 \text { for } z=z_{1} .
$$

The above-stated and the uniqueness of the solution of the thermoelasticity problems under consideration [2] result in the following theorem.

Theorem 2. The solution of a class of thermoelasticity boundary value problems (3), (5), (6), (7), (8), (15) for an SFAC consisting of a medium characterized by nine parameters $\left(c_{1}(z), c_{2}(z), \ldots \lambda_{2}(z)\right)$ satisfying restrictions $(4)$ is reduced to the integration of the system of differential equations

$$
\Delta_{2} T+\frac{1}{\lambda_{1}} \frac{\partial}{\partial z}\left(\lambda_{2} \frac{\partial T}{\partial z}\right)=0, \quad \Delta \varphi_{33}+c \frac{\partial^{2} \varphi_{33}}{\partial z^{2}}=\frac{k_{10}}{c_{1}} T
$$

when on each of the surfaces $\rho=\rho_{j}$ and $\alpha=\alpha_{j}$ the functions $T$ and $\varphi_{33}$ or their normal derivatives are assumed to be equal to zero and on each side $z=z_{j}$ one of conditions (8) and condition $\varphi_{33}=0$ of $\frac{\partial \varphi_{33}}{\partial z}=0$ are satisfied.

Note that a non-homogeneity class exists, i.e., a collection of functions $c_{1}(z)$, $c_{2}(z), \ldots, \lambda_{2}(z)$, for which $\varphi_{33}^{*}$ can be analytically found.

\section{Thermoelastic Equilibrium of Multilayer SFAC with Zero $\operatorname{rot} \vec{U}$ AND $D$}

Consider an SFAC multilayer along $z$, occupying the domain $\Omega_{z}=\Omega_{z 1}+$ $\Omega_{z 2}+\ldots+\Omega_{z \beta}$, where $\Omega_{z j}=\left\{\rho_{0}<\rho<\rho_{1}, \alpha_{0}<\alpha<\alpha_{1}, z_{j-1}<z<z_{j}\right\}$, $j=1,2, \ldots, \beta$, and $\beta$ is the number of layers. The layers $\Omega_{z 1}, \Omega_{z 2}, \ldots, \Omega_{z \beta}$ contact with one another along the planes $z=z_{j}$, where $j=1,2, \ldots, \beta-1$. Each layer has its elastic and thermal characteristics.

With $\rho=\rho_{j}(j=0,1)$ for all layers simultaneously conditions (6) are satisfied while with $\alpha=\alpha_{j}$ we have conditions (7). On the remaining boundary surfaces of the domain $\Omega_{z}$ conditions (8) and (15) are satisfied with $z_{1}$ substituted by $z_{\beta}$. On the contact plane $z=z_{j}\left(z=z_{j}\right.$ is the contact plane of the $j$-th and $j+1$-th layers) put

$$
\begin{gathered}
T_{j}-T_{j+1}=0, \quad \lambda_{2 j} \frac{\partial T_{j}}{\partial z}-\lambda_{2(j+1)} \frac{\partial T_{j+1}}{\partial z}=0, \\
\varphi_{33}^{(j)}-\varphi_{33}^{(j+1)}=0, \quad \frac{\partial \varphi_{33}^{(j)}}{\partial z}-\frac{\partial \varphi_{33}^{(j+1)}}{\partial z}=0 .
\end{gathered}
$$

The last two equalities, taking formulas (16) and (18) into account, imply the following contact conditions

$$
\begin{gathered}
u_{j}-u_{j+1}=0, \quad v_{j}-v_{j+1}=0, \quad w_{j}-w_{j+1}=0, \\
Z_{\rho j}-Z_{\rho(j+1)}=0, \quad Z_{\alpha j}-Z_{\alpha(j+1)}=0, \quad Z_{z j}-Z_{z(j+1)}=0 .
\end{gathered}
$$

The considerations presented in this section and the uniqueness of the solution of the boundary value contact problems given here [2] lead to the following theorem. 
Theorem 3. Consider a multilayer along the coordinate z SFAC, whose each $\beta$ layer is an SFAC of Theorem 2; furthermore, let the conditions on the surface of multilayer SFAC coincide with the conditions on the surface of the $S F A C$ from Theorem 2, the contact between the layers being rigid. In the case the solution to the boundary value contact problem is reduced to the integration of the equations

$$
\Delta T+\frac{1}{\lambda_{1 j}} \frac{\partial}{\partial z}\left(\lambda_{2 j} \frac{\partial T}{\partial z}\right)=0, \quad \Delta \varphi_{33}^{(j)}+c_{j} \frac{\partial^{2} \varphi_{33}^{(j)}}{\partial z^{2}}=\frac{k_{10}^{(j)}}{c_{1 j}} T_{j}, \quad j=1,2, \ldots, \beta,
$$

with the boundary conditions for $T_{j}$ and $\varphi_{33}^{(j)}$ coinciding, respectively, with boundary conditions $T$ and $\varphi_{33}$ in Theorem 2 and the contact conditions with respect to the functions $T_{j}, T_{j+1}, \varphi_{33}^{(j)}$ and $\varphi_{33}^{(j+1)}$ given in the second paragraph of this section.

It can be easily seen that in the case of the multilayer CPP under consideration we have $\operatorname{rot} \vec{U}=0$ and $D=0$.

\section{Thermoelastic Equilibrium of a Weakly Transversally Isotropic Homogeneous SFAC With $\operatorname{rot} \vec{U}=0$ AND $D=0$}

For $\lambda_{1}=$ const and $\lambda_{2}=$ const heat conductivity equation (3) will take the following form

$$
\Delta_{2} T+\lambda_{0} \frac{\partial^{2} T}{\partial z^{2}}=0
$$

where $\lambda_{0}=\frac{\lambda_{2}}{\lambda_{1}}$. In this case, using the method of separation of variables, the function $T$ in the domain $\Omega$ can be represented as

$$
T=a_{0}+b_{0} z+\sum_{n=0}^{\infty} \sum_{m=0}^{\infty}\left[A_{3 m n} e^{-p_{31} z}+B_{3 m n} e^{p_{31}\left(z-z_{1}\right)}\right] \psi_{m n}(\rho, \alpha),
$$

where $a_{0}, b_{0}, p_{31}(m, n)=\frac{p(m, n)}{\sqrt{\lambda_{0}}}>0, A_{m n}, B_{m n}$ are constant; $\psi_{m n}(\rho, \alpha)$ is a non-trivial solution of the following regular Sturm-Liouville problem [1]

$$
\begin{gathered}
\Delta_{2} \psi_{m n}+p^{2} \psi_{m n}=0 \\
\text { a) } \psi_{m n}=0 \text { or } \quad \text { b) } \frac{\partial \psi_{m n}}{\partial \rho}=0 \text { for } \rho=\rho_{j} \\
\text { a) } \psi_{m n}=0 \text { or } \quad \text { b) } \frac{\partial \psi_{m n}}{\partial \alpha}=0 \text { for } \alpha=\alpha_{j}
\end{gathered}
$$

Conditions (21) and (22) follow from conditions (6) and (7). Comments to the function $\psi_{m n}$ are exactly the same as in [2] to a similar function.

Although this section deals with a homogeneous SFAC, it is noteworthy that if $\lambda_{1}=\lambda_{1}(z)$ and $\lambda_{2}=\lambda_{2}(z)$, then

$$
T=\eta_{0}(z)+\sum_{n=0}^{\infty} \sum_{m=0}^{\infty} \eta_{m n}(z) \psi_{m n}(\rho, \alpha)
$$


where $\eta_{0}(z)$ and $\eta_{m n}(z)$ are the solution of the equations

$$
\frac{d}{d z}\left(\lambda_{2} \frac{d \eta_{0}}{d z}\right)=0 \text { and } \frac{1}{\lambda} \frac{d}{d z}\left(\lambda_{2} \frac{d \eta_{m n}}{d z}\right)-p^{2} \eta_{m n}=0
$$

Going back to the homogeneous SFAC, when all the nine parameters of the medium are constant we assume that

$$
\text { a) } T=\frac{c_{1}\left(\lambda_{0}-c\right)}{k_{10}} \frac{\partial^{2} T_{31}}{\partial z^{2}} \text { or b) } T=\frac{2 c c_{1}}{k_{10}} \frac{\partial T_{32}}{\partial z}
$$

which depends on whether $c \neq \lambda_{0}$ or $c=\lambda_{0}$. In (23a) we have

$$
\begin{aligned}
T_{31}= & \frac{a_{0}}{2}\left(z^{2}-\frac{\lambda_{0}}{2} r^{2}\right)+\frac{b_{0}}{2}\left(z^{3}-\frac{3 \lambda_{0}}{2} z \cdot r^{2}\right) \\
& +\sum_{n=0}^{\infty} \sum_{m=0}^{\infty}\left[A_{3 m n} e^{-p_{31} z}+B_{3 m n} e^{p_{31}\left(z-z_{1}\right)}\right] \frac{\psi_{m n}(\rho, \alpha)}{p_{31}^{2}} .
\end{aligned}
$$

The function $T_{32}$ can be represented by means of a series similar to $T_{31} ; r=$ $\sqrt{x^{2}+y^{2}}\left(x, y\right.$ are Cartesian coordinates). Obviously $T_{31}$ and $T_{32}$ satisfy the same equation as $T$.

For $c \neq \lambda_{0}$ the solution of (17) can be written as

$$
\varphi_{33}=\varphi_{33}^{0}+T_{31}
$$

where $\Delta_{2} T_{31}+\lambda_{0} \frac{\partial^{2} T_{31}}{\partial z^{2}}=0$, and for $c=\lambda_{0}$ the solution of (17) is expressed as

$$
\varphi_{33}=\varphi_{33}^{0}+z T_{32}
$$

where $\Delta_{2} T_{32}+c \frac{\partial^{2} T_{32}}{\partial z^{2}}=0$. Remember that in (25) and (26) $\varphi_{33}^{0}$ is the solution of (17) with a zero right-hand side.

For the function $\varphi_{33}^{0}$, similar to the function $T_{31}$, we have

$$
\begin{aligned}
\varphi_{33}^{0}= & a_{1}\left(2 z^{2}-c \cdot r^{2}\right)+b_{1}\left(2 z^{3}-3 c \cdot z \cdot r^{2}\right) \\
& +\sum_{n=0}^{\infty} \sum_{m=0}^{\infty}\left[C_{3 m n} e^{-p_{32} z}+D_{3 m n} e^{p_{32}\left(z-z_{1}\right)}\right] \psi_{m n}(\rho, \alpha),
\end{aligned}
$$

where $a_{1}, b_{1}, p_{32}(m, n)=\frac{p(m, n)}{\sqrt{c}}, C_{m n}, D_{m n}$ are constant.

Formulas (23)-(27) suggest that boundary value problems (5), (6), (7), (8), (15) have been solved for a weakly transversally isotropic homogeneous SFAC. We can illustrate it by the solution of boundary value problem (5), (6b), (7b), (8a), (15b) for $c \neq \lambda_{0}$ applying the Fourier method.

Similar to [1], for $T_{31}$ we have

$$
\begin{aligned}
T_{31}=\sum_{n=1}^{\infty} \sum_{m=1}^{\infty}\left(\frac{\tau_{30 m n}-e^{-p_{31} z} \cdot \tau_{31 m n}}{1-e^{-2 p_{31} z_{1}}} e^{-p_{31} z}\right. \\
\left.\quad+\frac{\tau_{31 m n}-e^{-p_{31} z} \cdot \tau_{30 m n}}{1-e^{-2 p_{31} z_{1}}} e^{p_{31}\left(z-z_{1}\right)}\right) \psi_{m n}(\rho, \alpha) .
\end{aligned}
$$


In $(28) \tau_{30 m n}$ and $\tau_{31 m n}$ are Fourier coefficients of the functions $\tau_{30}(\rho, \alpha)$ and $\tau_{31}(\rho, \alpha)$ expanded into a Fourier series with respect to the functions $\psi_{m n}$, where the functions $\psi_{m n}$ are a non-trivial solution of problem (20), (21a), (22a).

In the expression for the function

$$
\varphi_{33}^{0}=\sum_{n=1}^{\infty} \sum_{m=1}^{\infty}\left[C_{3 m n} e^{-p_{32} z}+D_{3 m n} e^{p_{32}\left(z-z_{1}\right)}\right] \psi_{m n}(\rho, \alpha)
$$

the constants $C_{3 m n}$ and $D_{3 m n}$ with formula (25) and boundary conditions (19a) in mind (conditions (19a) lead to conditions (15b)) are established using the following system of equations

$$
\begin{aligned}
C_{3 m n}+e^{-p_{32} z_{1}} D_{3 m n} & =-\tau_{30 m n}, \\
e^{-p_{32}} C_{3 m n}+D_{3 m n} & =-\tau_{31 m n} .
\end{aligned}
$$

The determinant of this system $1-e^{-2 p_{32} z_{1}}$ and the expression $1-e^{-2 p_{31} z_{1}}$ in (28) exceed zero since $p_{32}>0$ and $p_{31}>0$.

Having determined $C_{3 m n}$ and $D_{3 m n}$ from system (30) and substituting the obtained solution into (29) we shall have the solution of the stated problem. The uniqueness of the obtained solution and convergence of the corresponding series can be easily proved [2].

The analytical solution of any of boundary value problems (5), (6), (7), (8), (15) can be constructed in a similar way both for $c \neq \lambda_{0}$ and $c=\lambda_{0}$.

\section{Weakly Transversally Isotropic Rectangular Parallelepiped}

In the given section it is assumed that besides condition (4a) and (4b) there is also the following condition

$$
c=g \text {. }
$$

With (31) in mind, in Cartesian coordinates $x, y, z$, with constant elastic and thermal characteristics of the medium system (5) can be written as
a) $c \frac{\partial D}{\partial z}-\frac{\partial K_{y}}{\partial x}+\frac{\partial K_{x}}{\partial y}=0$
e) $\frac{\partial u}{\partial x}+\frac{\partial v}{\partial y}+c \frac{\partial w}{\partial z}-\frac{k_{10}}{c_{1}} T=\frac{D}{c_{1}}$,
b) $\frac{\partial D}{\partial x}-\frac{\partial K_{z}}{\partial y}+\frac{\partial K_{y}}{\partial z}=0$,
f) $\frac{\partial w}{\partial y}-\frac{\partial v}{\partial z}=\frac{K_{x}}{c_{4}}$
c) $\frac{\partial D}{\partial y}-\frac{\partial K_{x}}{\partial z}+\frac{\partial K_{z}}{\partial x}=0$
g) $\frac{\partial u}{\partial z}-\frac{\partial w}{\partial x}=\frac{K_{y}}{c_{4}}$
d) $\frac{\partial K_{x}}{\partial x}-\frac{\partial K_{y}}{\partial y}+c \frac{\partial K_{z}}{\partial z}=0$
h) $\frac{\partial v}{\partial x}-\frac{\partial u}{\partial y}=\frac{K_{z}}{c_{5}}$.

We can express the solution of system (32) by means of functions $\varphi_{21}, \varphi_{22}$, $\varphi_{23}$, where $\operatorname{frac} \partial^{2} \varphi_{2 i} \partial x^{2}+\frac{\partial^{2} \varphi_{2 i}}{\partial y^{2}}+c \frac{\partial^{2} \varphi_{2 i}}{\partial z^{2}}=\Delta_{c} \varphi_{2 i}=0(i=1,2,3)$, assuming that the surface and thermal disturbances are defined on the sides $x=0$ and $x=x_{1}$ or $y=0$ and $y=y_{1}$ of a rectangular parallelepiped (RP) occupying the domain $\Omega=\left\{0<x<x_{1}, 0<y<y_{1}, 0<z<z_{1}\right\}$ ( $z=$ const remains to be the plane of isotropy of the medium). As an illustration we are going to consider in 
detail the case when the disturbance is defined on the sides $y=0$ and $y=y_{1}$, or to be more exact, when we have the following boundary conditions:

For $z=z_{j}$ we have : a) $\frac{\partial T}{\partial z}=0, \quad w=0, \quad X_{z}=0, Y_{z}=0 \quad$ or

b) $T=0, \quad Z_{z}=0, \quad u=0, \quad v=0$.

For $x=x_{j}$ we have : a) $\frac{\partial T}{\partial x}=0, \quad u=0, \quad Y_{x}=0, \quad Z_{x}=0 \quad$ or

b) $T=0, \quad X_{x}=0, \quad v=0, \quad w=0$.

For $y=y_{j}$ we have : a) $T=\tau_{2 j}(z, x)$ or b) $\frac{\partial T}{\partial y}=\widetilde{\tau}_{2 j}(z, x)$ or

c) $\frac{\partial T}{\partial y}+\theta_{2 j} T=\widetilde{\tau}_{2 j}(z, x)$.

For $y=y_{j}$ we have : $q_{j 1} Y_{y}+q_{j 2} v=F_{2 j 1}(z, x)$,

$$
\begin{aligned}
& q_{j 3} Z_{y}+q_{j 4} w=F_{2 j 2}(z, x), \\
& q_{j 5} X_{y}+q_{j 6} u=F_{2 j 3}(z, x) .
\end{aligned}
$$

In $(33)-(36) j=0,1, z_{0}=0, x_{0}=0, y_{0}=0 ; q_{j i}$ and $q_{j(i+1)}$ are constant with $q_{j i} \cdot q_{j(i+1)} \geq 0(i=1,3,5)$; the requirements to the functions $\tau_{2 j}(z, x), \widetilde{\tau}_{2 j}(z, x)$, $F_{2 j 1}(z, x), \ldots, F_{2 j s}(z, x)$ are similar to the requirements to the functions appearing in (8) and (9). Conditions (33a) and (34a) are called symmetry conditions while conditions (33b) and (34b) are called antisymmetry conditions. Note that conditions (9) can also be replaced by more general conditions of type (36) if the domain $\Omega$ implied a rectangular parallelepiped.

Let

$$
D=\frac{\partial \varphi_{21}}{\partial y}
$$

then $(32 \mathrm{a}, \mathrm{b}, \mathrm{c})$ can be expressed in the following way

$$
\begin{aligned}
& \frac{\partial}{\partial y}\left(K_{x}+c \frac{\partial \varphi_{21}}{\partial z}\right)-\frac{\partial K_{y}}{\partial x}=0 \\
& \frac{\partial K_{y}}{\partial z}-\frac{\partial}{\partial y}\left(K_{z}-\frac{\partial \varphi_{21}}{\partial x}\right)=0 \\
& \frac{\partial}{\partial x}\left(K_{z}-\frac{\partial \varphi_{21}}{\partial x}\right)-\frac{\partial}{\partial z}\left(K_{x}+c \frac{\partial \varphi_{21}}{\partial z}\right)=0
\end{aligned}
$$

The latter equalities imply that a function $\frac{\partial \varphi_{22}}{\partial y}$ exists, such that

$$
K_{x}=\frac{\partial^{2} \varphi_{22}}{\partial x \partial y}-c \frac{\partial \varphi_{21}}{\partial z}, \quad K_{y}=\frac{\partial^{2} \varphi_{22}}{\partial y^{2}}, \quad K_{z}=\frac{\partial^{2} \varphi_{22}}{\partial z \partial y}+\frac{\partial \varphi_{21}}{\partial x}
$$

Substituting (37) into (32d) we obtain
a) $\frac{\partial}{\partial y} \Delta_{c} \varphi_{22}=0 \quad$ or
b) $\Delta_{c} \varphi_{22}=f(z, x)$, 
where $f(z, x)$ is a function resulting from the integration of equation (38a). Similar to the case of equation $(13 b)$, it can be shown that $f(z, x)=0$.

Equations $(32 \mathrm{e}, \mathrm{f}, \mathrm{g}, \mathrm{h})$, taking into account the expressions for $D$, formulas (37) and condition (31), can be written as

$$
\begin{aligned}
& \frac{\partial u}{\partial x}+\frac{\partial v}{\partial y}+c \frac{\partial w}{\partial z}=\frac{1}{c_{1}} \frac{\partial \varphi_{21}}{\partial y}+\frac{k_{10}}{c_{1}} T, \\
& \frac{\partial}{\partial y}\left(w-\frac{1}{c_{4}} \frac{\partial \varphi_{22}}{\partial x}\right)-\frac{\partial}{\partial z}\left(v-\frac{\varphi_{21}}{c_{5}}\right)=0, \\
& \frac{\partial}{\partial z}\left(u+\frac{1}{c_{5}} \frac{\partial \varphi_{22}}{\partial z}\right)-\frac{\partial}{\partial x}\left(w-\frac{1}{c_{4}} \frac{\partial \varphi_{22}}{\partial x}\right)=0, \\
& \frac{\partial}{\partial x}\left(v-\frac{\varphi_{21}}{c_{5}}\right)-\frac{\partial}{\partial y}\left(u+\frac{1}{c_{5}} \frac{\partial \varphi_{22}}{\partial z}\right)=0,
\end{aligned}
$$

It follows from (39) that

$$
u=\frac{\partial \bar{\varphi}_{23}}{\partial x}-\frac{1}{c_{5}} \frac{\partial \varphi_{22}}{\partial z}, \quad v=\frac{\partial \bar{\varphi}_{23}}{\partial y}+\frac{\varphi_{21}}{c_{5}}, \quad w=\frac{\partial \bar{\varphi}_{23}}{\partial z}+\frac{1}{c_{4}} \frac{\partial \varphi_{22}}{\partial x}
$$

where

$$
\Delta_{c} \bar{\varphi}_{23}=\frac{c_{5}-c_{1}}{c_{1} c_{5}} \frac{\partial \varphi_{21}}{\partial y}+\frac{k_{10}}{c_{1}} T
$$

For $c \neq \lambda_{0}$ let

$$
T=\frac{c_{1}\left(\lambda_{0}+c\right)}{k_{10}} \frac{\partial^{2} T_{21}}{\partial z^{2}}
$$

where $\Delta_{2} T_{21}+\lambda_{0} \frac{\partial^{2} T_{21}}{\partial z^{2}}=0$ and for $c=\lambda_{0}$ let

$$
T=\frac{2 c_{1}}{k_{10}} \frac{\partial T_{22}}{\partial y}
$$

where $\Delta_{c} T_{22}=0$, then for $c \neq \lambda_{0}$ the solution of equation (41) will be

$$
\bar{\varphi}_{23}=\varphi_{23}+\frac{c_{5}-c_{1}}{2 c_{1} c_{5}} y \varphi_{21}+T_{21}
$$

while for $c=\lambda$ we shall have

$$
\bar{\varphi}_{23}=\varphi_{23}+\frac{c_{5}-c_{1}}{2 c_{1} c_{5}} y \varphi_{21}+y T_{22} .
$$

If we substitute the expression $\bar{\varphi}_{33}$ obtained for $c \neq \lambda_{0}$ into (40) we have

$$
\begin{aligned}
& u=\frac{\partial}{\partial x}\left(\varphi_{23}+\frac{c_{5}-c_{1}}{2 c_{1} c_{5}} y \varphi_{21}\right)-\frac{1}{c_{5}} \frac{\partial \varphi_{22}}{\partial z}+\frac{\partial T_{21}}{\partial x} \\
& v=\frac{\partial}{\partial y}\left(\varphi_{23}+\frac{c_{5}-c_{1}}{2 c_{1} c_{5}} y \varphi_{21}\right)+\frac{\varphi_{21}}{c_{5}}+\frac{\partial T_{21}}{\partial y} \\
& w=\frac{\partial}{\partial z}\left(\varphi_{23}+\frac{c_{5}-c_{1}}{2 c_{1} c_{5}} y \varphi_{21}\right)+\frac{1}{c_{4}} \frac{\partial \varphi_{22}}{\partial x}+\frac{\partial T_{21}}{\partial z}
\end{aligned}
$$


If in the expressions for $u, v$ and $w$ the last members, i.e., $\frac{\partial T_{21}}{\partial x}, \frac{\partial T_{21}}{\partial y}$ and $\frac{\partial T_{21}}{\partial z}$ are substituted, respectively, by $y \frac{\partial T_{22}}{\partial x}, \frac{\partial}{\partial y}\left(y T_{22}\right)$ and $\frac{\partial T_{22}}{\partial z}$, we shall obtain a solution of system (33) represented by functions $\varphi_{21}, \varphi_{22}$ and $\varphi_{23}$ for $c=\lambda_{0}$.

When a solution of system (32) expressed by formulas (42) is available, we can construct analytically (in series) the solution of any boundary value problems (32), (33), (34), (35), (36) as well as of the corresponding boundary valuecontact problems for a multilayer RP both for $c \neq \lambda_{0}$ and $c=\lambda_{0}$. The technique is similar to that used in [2] by the given author. The above stated implies the following: system (32) can be analytically solved taking into consideration boundary conditions (33), (34) and

$$
\begin{gathered}
\left.\frac{\partial T}{\partial y}\right|_{y=y_{j}}=\widetilde{\tau}_{2 j}(x, z),\left.\quad v\right|_{y=y_{j}}=f_{2 j 1}(x, z), \\
\left.Z_{y}\right|_{y=y_{j}}=F_{2 j 2}(x, z),\left.\quad X_{y}\right|_{y=y_{j}}=F_{2 j 3}(x, z)
\end{gathered}
$$

or

$$
\begin{aligned}
& \left.T\right|_{y=y_{j}}=\tau_{2 j}(x, z),\left.\quad Y_{y}\right|_{y=y_{j}}=F_{2 j 1}(x, z), \\
& \left.w\right|_{y=y_{j}}=f_{2 j 2}(x, z),\left.\quad u\right|_{y=y_{j}}=f_{2 j 3}(x, z) .
\end{aligned}
$$

If the above is true, then summing up the solutions we can also solve system (32) analytically taking into account both nonhomogeneous boundary conditions (33), (34) (in the right-hand sides of equalities (33) and (34) there are defined functions instead of zero) and the boundary conditions which appear in the previous sentence. Without dwelling on it consider the case when $\varphi_{21}=0$ and $\varphi_{22}=0$.

\section{Equilibrium of RP with Thermal Disturbance on the Sides

$$
y=y_{j} \text { OR } x=x_{j} \text { WHEN } \operatorname{rot} \vec{U}=0 \text { AND } D=0
$$

Let $\varphi_{21}=0$ and $\varphi_{22}=0$, i.e., $D=0$ and $\operatorname{rot} \vec{U}=0$ or $D=0, K_{x}=0$, $K_{y}=0, K_{z}=0$, then for $c \neq \lambda_{0}$ (42) implies

$$
u=\frac{\partial}{\partial x}\left(\varphi_{23}+T_{21}\right), \quad v=\frac{\partial}{\partial y}\left(\varphi_{23}+T_{21}\right), \quad w=\frac{\partial}{\partial z}\left(\varphi_{23}+T_{21}\right) .
$$

It should be noted that for $D=0, K_{\rho}=0, K_{\alpha}=0$ and $K_{z}=0$ representation (43) and all other representations considered in this section remain true for $c \neq g$ as well. In other words, neither the condition $c \neq g$ nor $c=g$ will in any way affect either (43) or other representations similar to (43).

If $c=\lambda_{0}$, then

$$
u=\frac{\partial \varphi_{23}}{\partial x}+y \frac{\partial T_{22}}{\partial x}, \quad v=\frac{\partial \varphi_{23}}{\partial y}+y \frac{\partial T_{22}}{\partial y}+T_{22}, \quad w=\frac{\partial \varphi_{23}}{\partial z}+y \frac{\partial T_{22}}{\partial z} .
$$

By means of representations (43) and (44) the thermoelastic equilibrium of a weakly transversally isotropic RP can be found under boundary conditions 
(33), (34), (35) and

$$
\begin{array}{ll}
\text { a) }\left.v\right|_{y=y_{j}}=0,\left.\quad Z_{y}\right|_{y=y_{j}}=0,\left.\quad Z_{x}\right|_{y=y_{j}}=0, \quad \text { or } \\
\text { b) }\left.Y_{y}\right|_{y=y_{j}}=0,\left.\quad w\right|_{y=y_{j}}=0,\left.\quad u\right|_{y=y_{j}}=0 .
\end{array}
$$

The thermoelastic equilibrium of an RP under boundary conditions (33), $(34)^{\prime}$ (a prime over the number means that in (34a) the condition $\frac{\partial T}{\partial x}=0$ is omitted while in (34b) we omit the condition $T=0$ ), $(45)^{\prime}$ (a prime over the number means that (45a) is supplemented by the condition $\left.\frac{\partial T}{\partial y}\right|_{y=y_{j}}=0$ and (45b) is supplemented by the condition $\left.T\right|_{y=y_{j}}=0$ ) and

$$
\begin{aligned}
& \text { a) }\left.T\right|_{x=\overline{\overline{x_{j}}}} \tau_{1 j}(y, z) \text { or b) }\left.\frac{\partial T}{\partial x}\right|_{x=x_{j}}=\widetilde{\tau}_{1 j}(y, z) \text {, or } \\
& \text { c) }\left(\frac{\partial T}{\partial x}+\theta_{1 j} T\right)_{x=x_{j}}=\widetilde{\tau}_{1 j}(y, z),
\end{aligned}
$$

for $c \neq \lambda_{0}$ can be represented as

$$
u=\frac{\partial}{\partial x}\left(\varphi_{13}+T_{11}\right), \quad v=\frac{\partial}{\partial y}\left(\varphi_{13}+T_{11}\right), \quad w=\frac{\partial}{\partial z}\left(\varphi_{13}+T_{11}\right)
$$

where $\Delta_{c} \varphi_{13}=0, \Delta_{2} T_{11}+\lambda_{0} \frac{\partial^{2} T_{11}}{\partial z^{2}}=0, T=\frac{c_{1}\left(\lambda_{0}+c\right)}{k_{10}} \frac{\partial^{2} T_{11}}{\partial z^{2}}$ and for $c=\lambda_{0}$ as

$$
u=\frac{\partial \varphi_{13}}{\partial x}+x \frac{\partial T_{12}}{\partial x}+T_{12}, \quad v=\frac{\partial \varphi_{13}}{\partial y}+x \frac{\partial T_{12}}{\partial y}, \quad w=\frac{\partial \varphi_{13}}{\partial z}+x \frac{\partial T_{12}}{\partial z},
$$

where $\Delta_{c} T_{12}=0, T=\frac{2 c_{1}}{k_{10}} \frac{\partial T_{12}}{\partial x}$.

\section{Equilibrium of RP with Thermal Disturbance on the Six Sides WHEN $\operatorname{rot} \vec{U}=0$ AND $D=0$}

Construct now for the RP the solution of boundary value problems (32), (34)*, $(45)^{*},(33)^{*}$, where $(34)^{*}$, means that in (34a) instead the condition $\left.\frac{\partial T}{\partial x}\right|_{x=x_{j}}=0$ we have the condition $\left.\frac{\partial T}{\partial x}\right|_{x=x_{j}}=\widetilde{\tau}_{1 j}(y, z)$ and in $(34 b)$ instead of the condition $\left.T\right|_{x=x_{j}}=0$ we have the condition $\left.T\right|_{x=x_{j}}=\tau_{1 j}(y, z) ;(45)^{*}$ means that $(45 a)$ is supplemented by the condition $\left.\frac{\partial T}{\partial y}\right|_{y=y_{j}}=\widetilde{\tau}_{2 j}(z, x)$ and (45b) by the condition $\left.T\right|_{y=y_{j}}=\tau_{2 j}(z, x) ;(33)^{*}$ means that in $(33 a)$ instead of the condition $\left.\cdot \frac{\partial T}{\partial z}\right|_{z=z_{j}}=$ 0 we take the condition $\left.\cdot \frac{\partial T}{\partial z}\right|_{z=z_{j}}=\widetilde{\tau}_{3 j}(x, y)$ and in $(33 b)$ instead of the condition $\left.T\right|_{z=z_{j}}=0$ we take the condition $\left.T\right|_{z=z_{j}}=\tau_{3 j}(x, y)$. We shall also assume that the compatibility conditions on the edges of the RP are homogeneous (if the compatibility conditions are non-homogeneous they can be reduced to homogeneous conditions using the technique of [5]).

The solution of boundary value problems $(32),(34)^{*},(45)^{*},(33)^{*}$ is constructed by means of summation of the solutions of boundary value problems (32), $(34)^{*},(45)^{\prime},(33)$; (32), (34), (45)* , (33); (32), (34), (45)', (33)*. 
As an example, using the method of separation of variables, we can construct the solution of boundary value problem $(32),(34 b)^{*},(45 b)^{*},(33 b)^{*}$ for $c \neq \lambda_{0}$ representing it as a sum of the solutions of boundary value problems (32), $(34 \mathrm{~b})^{*},(45 \mathrm{~b})^{\prime},(33 \mathrm{~b}) ;(32),(34 \mathrm{~b}),(45 \mathrm{~b})^{*},(33 \mathrm{~b}) ;(32),(34 \mathrm{~b}),(45 \mathrm{~b})^{\prime},(33 \mathrm{~b})^{*}$. If we denote the components of the displacement vector by $u_{1}, v_{1}$ and $w_{1}$ in the case of boundary value problem $(32),(34 \mathrm{~b})^{*},(45 \mathrm{~b}),(33 \mathrm{~b})$, by $u_{2}, v_{2}$ and $w_{2}$ in the case of boundary value problem $(32),(34 \mathrm{~b}),(45 \mathrm{~b})^{*},(33 \mathrm{~b})$, by $u_{3}, v_{3}$ and $w_{3}$ in the case of boundary value problem $(32),(34 \mathrm{~b}),(45 \mathrm{~b})^{\prime},(33 \mathrm{~b})^{*}$ and by $u, v$ and $w$ in the case of boundary value problem $(32),(34 \mathrm{~b})^{*},(45 \mathrm{~b})^{*},(33 \mathrm{~b})^{*}$, then

$$
u=u_{1}+u_{2}+u_{3}, \quad v=v_{1}+v_{2}+v_{3}, \quad w=w_{1}+w_{2}+w_{3} .
$$

In equality (49) we have
a) $u_{1}=\frac{\partial}{\partial x}\left(\varphi_{13}+T_{11}\right), \quad v_{1}=\frac{\partial}{\partial y}\left(\varphi_{13}+T_{11}\right), \quad w_{1}=\frac{\partial}{\partial z}\left(\varphi_{13}+T_{11}\right)$;
b) $u_{2}=\frac{\partial}{\partial x}\left(\varphi_{23}+T_{21}\right), \quad v_{2}=\frac{\partial}{\partial y}\left(\varphi_{23}+T_{21}\right), \quad w_{2}=\frac{\partial}{\partial z}\left(\varphi_{23}+T_{21}\right)$;
c) $u_{3}=\frac{\partial}{\partial x}\left(\varphi_{33}+T_{31}\right), \quad v_{3}=\frac{\partial}{\partial y}\left(\varphi_{33}+T_{31}\right), \quad w_{3}=\frac{\partial}{\partial z}\left(\varphi_{33}+T_{31}\right)$;

In $(50)$ we have

$$
\begin{aligned}
& T_{11}=\sum_{n=1}^{\infty} \sum_{m=1}^{\infty}\left[\frac{\tau_{10 m n}-e^{-p_{11} x_{1}} \tau_{11 m n}}{1-e^{-2 p_{11} x_{1}}} e^{-p_{11} x}+\frac{\tau_{11 m n}-e^{-p_{11} x_{1}} \tau_{10 m n}}{1-e^{-2 p_{11} x_{1}}} e^{p_{11}\left(x-x_{1}\right)}\right] \\
& \times \sin \left(\frac{\pi m}{y_{1}} y\right) \sin \left(\frac{\pi m}{z_{1}} z\right), \\
& \varphi_{13}=\sum_{n=1}^{\infty} \sum_{m=1}^{\infty}\left[\frac{e^{-p_{12} x_{1}} \tau_{11 m n}-\tau_{10 m n}}{1-e^{-2 p_{12} x_{1}}} e^{-p_{12} x}+\frac{e^{-p_{12} x_{1}} \tau_{10 m n}-\tau_{11 m n}}{1-e^{-2 p_{12} x_{1}}} e^{p_{12}\left(x-x_{1}\right)}\right] \\
& \times \sin \left(\frac{\pi m}{y_{1}} y\right) \sin \left(\frac{\pi m}{z_{1}} z\right) ; \\
& T_{21}=\sum_{n=1}^{\infty} \sum_{m=1}^{\infty}\left[\frac{\tau_{20 m n}-e^{-p_{21} y_{1}} \tau_{21 m n}}{1-e^{-2 p_{21} y_{1}}} e^{-p_{21} y}+\frac{\tau_{21 m n}-e^{-p_{21} y_{1}} \tau_{20 m n}}{1-e^{-2 p_{21} y_{1}}} e^{p_{21}\left(y-y_{1}\right)}\right] \\
& \times \sin \left(\frac{\pi m}{z_{1}} z\right) \sin \left(\frac{\pi m}{x_{1}} x\right), \\
& \varphi_{23}=\sum_{n=1}^{\infty} \sum_{m=1}^{\infty}\left[\frac{e^{-p_{22} y_{1}} \tau_{21 m n}-\tau_{20 m n}}{1-e^{-2 p_{22} y_{1}}} e^{-p_{22} y}+\frac{e^{-p_{22} y_{1}} \tau_{20 m n}-\tau_{21 m n}}{1-e^{-2 p_{22} y_{1}}} e^{p_{22}\left(y-y_{1}\right)}\right] \\
& \times \sin \left(\frac{\pi m}{z_{1}} z\right) \sin \left(\frac{\pi m}{x_{1}} x\right) \\
& T_{31}=\sum_{n=1}^{\infty} \sum_{m=1}^{\infty}\left[\frac{\tau_{30 m n}-e^{-p_{31} z_{1}} \tau_{31 m n}}{1-e^{-2 p_{31} z_{1}}} e^{-p_{31} z}+\frac{\tau_{31 m n}-e^{-p_{31} z_{1}} \tau_{30 m n}}{1-e^{-2 p_{31} z_{1}}} e^{p_{31}\left(z-z_{1}\right)}\right]
\end{aligned}
$$




$$
\begin{aligned}
& \times \sin \left(\frac{\pi m}{x_{1}} x\right) \sin \left(\frac{\pi m}{y_{1}} y\right) \\
& \varphi_{33}^{0}=\sum_{n=1}^{\infty} \sum_{m=1}^{\infty}\left[\frac{e^{-p_{32} z_{1}} \tau_{31 m n}-\tau_{30 m n}}{1-e^{-2 p_{32} z_{1}} e^{-p_{32} z}}+\frac{e^{-p_{32} z_{1}} \tau_{30 m n}-\tau_{31 m n}}{1-e^{-2 p_{32} z_{1}}} e^{p_{32}\left(z-z_{1}\right)}\right] \\
& \times \sin \left(\frac{\pi m}{x_{1}} x\right) \sin \left(\frac{\pi m}{y_{1}} y\right) .
\end{aligned}
$$

$\operatorname{In}(51)$

$$
\begin{aligned}
& p_{11}=\pi \sqrt{\left(\frac{m}{y_{1}}\right)^{2}+\lambda_{0}\left(\frac{n}{z_{1}}\right)^{2}}, \quad p_{12}=\pi \sqrt{\left(\frac{m}{y_{1}}\right)^{2}+c\left(\frac{n}{z_{1}}\right)^{2}}, \\
& p_{21}=\pi \sqrt{\lambda_{0}\left(\frac{m}{z_{1}}\right)^{2}+\left(\frac{n}{x_{1}}\right)^{2}}, \quad p_{22}=\pi \sqrt{c\left(\frac{m}{z_{1}}\right)^{2}+\left(\frac{n}{x_{1}}\right)^{2}}, \\
& p_{31}=\pi \sqrt{\frac{1}{\lambda_{0}}\left[\left(\frac{m}{x_{1}}\right)^{2}+\left(\frac{n}{y_{1}}\right)^{2}\right]}, \quad p_{32}=\pi \sqrt{\frac{1}{c}\left[\left(\frac{m}{x_{1}}\right)^{2}+\left(\frac{n}{y_{1}}\right)^{2}\right]} \text {; }
\end{aligned}
$$

$\tau_{10 m n}, \tau_{11 m n}, \tau_{20 m n}, \tau_{21 m n}, \tau_{30 m n}, \tau_{31 m n}$ are Fourier coefficients of the functions $\tau_{10}(y, z), \tau_{11}(y, z), \tau_{20}(z, x), \tau_{21}(z, x), \tau_{30}(x, y), \tau_{31}(x, y)$, respectively. The uniqueness of the obtained solution and convergence of the corresponding series can be easily proved.

Quite similarly one can construct a solution of any other boundary value problem from the class of boundary value problems $(32),(34)^{*},(45)^{*},(33)^{*}$ for an RP, both for $c \neq \lambda_{0}$ and $c=\lambda_{0}$ (mind that the medium constituting the RP is characterized by nine elastic and thermal constants correlated by means of conditions (4a) and (4b).

The above-stated suggests that the following Theorem has been proved.

Theorem 4. For a weakly transversally isotropic homogeneous RP the precise solution of boundary value problems $(32),(34)^{*},(45)^{*},(33)^{*}$; is constructed by summing up the solutions of boundary value problems $(32),(34)^{*},(45)^{\prime},(33)$; (32), (34), (45)*, (33); (32), (34), (45)', (33)* . Finally the components of the displacement vector and stress tensor are represented as double series of form (51).

Remark 1. If on each of the six sides of the RP the defined density of the thermal flow or the defined temperature (including the case when the temperature is defined for some sides while for other sides the density of the thermal flow is given) is represented as a finite double series, then the precise solution becomes elementary.

Remark 2. The boundary value problems considered in this section are of practical importance since the boundary conditions which appear in these problems may have a practical interpretation. In particular, boundary conditions 
$(34 \mathrm{~b})^{*},(45 \mathrm{~b})^{*}$ and $(33 \mathrm{~b})^{*}$ can be interpreted in the following way: thin nontensile and non-compressible plates are attached to the sides of an RP and in each plate the defined temperature distribution is maintained.

\section{APPENDIX}

Find the thermoelastic equilibrium of a homogeneous cube $\Omega=\{0<x<\pi$, $0<y<\pi, 0<z<\pi\}$, for $\lambda_{0}=2, c=1, g=1, c_{1}-2 c_{4}=c_{3}$ and $k_{1}=k_{2}$, with the following boundary conditions:

$$
\begin{aligned}
& X_{x}=0, \quad v=0, \quad w=0, \quad T=0 \text { for } x=0, \\
& X_{x}=0, \quad v=0, \quad w=0, \quad T=A_{1} \sin y \sin z \text { for } x=\pi ; \\
& Y_{y}=0, \quad w=0, \quad u=0, \quad T=0 \text { for } y=0, \\
& Y_{y}=0, \quad w=0, \quad u=0, \quad T=A_{2} \sin z \sin x \text { for } y=\pi ; \\
& Z_{z}=0, \quad u=0, \quad v=0, \quad T=0 \text { for } z=0, \\
& Z_{z}=0, \quad u=0, \quad v=0, \quad T=A_{3} \sin x \sin y \text { for } z=\pi,
\end{aligned}
$$

where $A_{i}$ are arbitrary constants $(i=1,2,3)$.

According to formulas (50), we have

$$
u=\frac{\partial \Phi}{\partial x}, \quad v=\frac{\partial \Phi}{\partial y}, \quad w=\frac{\partial \Phi}{\partial z},
$$

where $\Phi=\varphi_{13}+T_{11}+\varphi_{23}+T_{33}+\varphi_{33}^{0}+T_{33}$. As for $\varphi_{13}, T_{11}, \ldots, T_{33}$, it follows from formulas (51)

$$
\begin{array}{ll}
T_{11}=A_{1} \frac{\sinh (\sqrt{3} x)}{\sinh (\sqrt{3} \pi)} \sin y \sin z, & \varphi_{13}=-A_{1} \frac{\sinh (\sqrt{2} x)}{\sinh (\sqrt{2} \pi)} \sin y \sin z \\
T_{21}=A_{2} \frac{\sinh (\sqrt{3} y)}{\sinh (\sqrt{3} \pi)} \sin z \sin x, & \varphi_{23}=-A_{2} \frac{\sinh (\sqrt{2} y)}{\sinh (\sqrt{2} \pi)} \sin z \sin x \\
T_{31}=A_{3} \frac{\sinh z}{\sinh \pi} \sin x \sin y, & \varphi_{33}^{0}=-A_{3} \frac{\sinh (\sqrt{2} z)}{\sinh (\sqrt{2} \pi)} \sin x \sin y
\end{array}
$$

Using the function $\Phi$ we can express stresses as

$$
\begin{aligned}
\frac{1}{2 c_{4}} X_{x} & =-\left(\frac{\partial^{2} \Phi}{\partial y^{2}}+\frac{\partial^{2} \Phi}{\partial z^{2}}\right), & \frac{1}{2 c_{4}} Y_{y} & =-\left(\frac{\partial^{2} \Phi}{\partial x^{2}}+\frac{\partial^{2} \Phi}{\partial z^{2}}\right), \\
\frac{1}{2 c_{4}} Z_{z} & =-\left(\frac{\partial^{2} \Phi}{\partial x^{2}}+\frac{\partial^{2} \Phi}{\partial y^{2}}\right), & \frac{1}{2 c_{4}} Z_{x} & =\frac{\partial^{2} \Phi}{\partial x \partial z} \\
\frac{1}{2 c_{4}} Z_{y} & =\frac{\partial^{2} \Phi}{\partial y \partial z}, & \frac{1}{2 c_{4}} Y_{x} & =\frac{\partial^{2} \Phi}{\partial x \partial y} .
\end{aligned}
$$

In conclusion, we shall give some graphs describing changes in the displacements $u$ and $w$ and the stresses $X_{x}, Z_{z}, Y_{x}$ and $Z_{x}$ along the diagonal of the cube passing through the points $M_{1}(0,0,0)$ and $M_{2}(\pi, \pi, \pi)$ for $A_{1}=A_{2}=A_{3}=1$ (see Fig. 1). It should be noted that on the diagonal $M_{1} M_{2}$ we have $X_{x}=$ $Y_{y}=0$. 

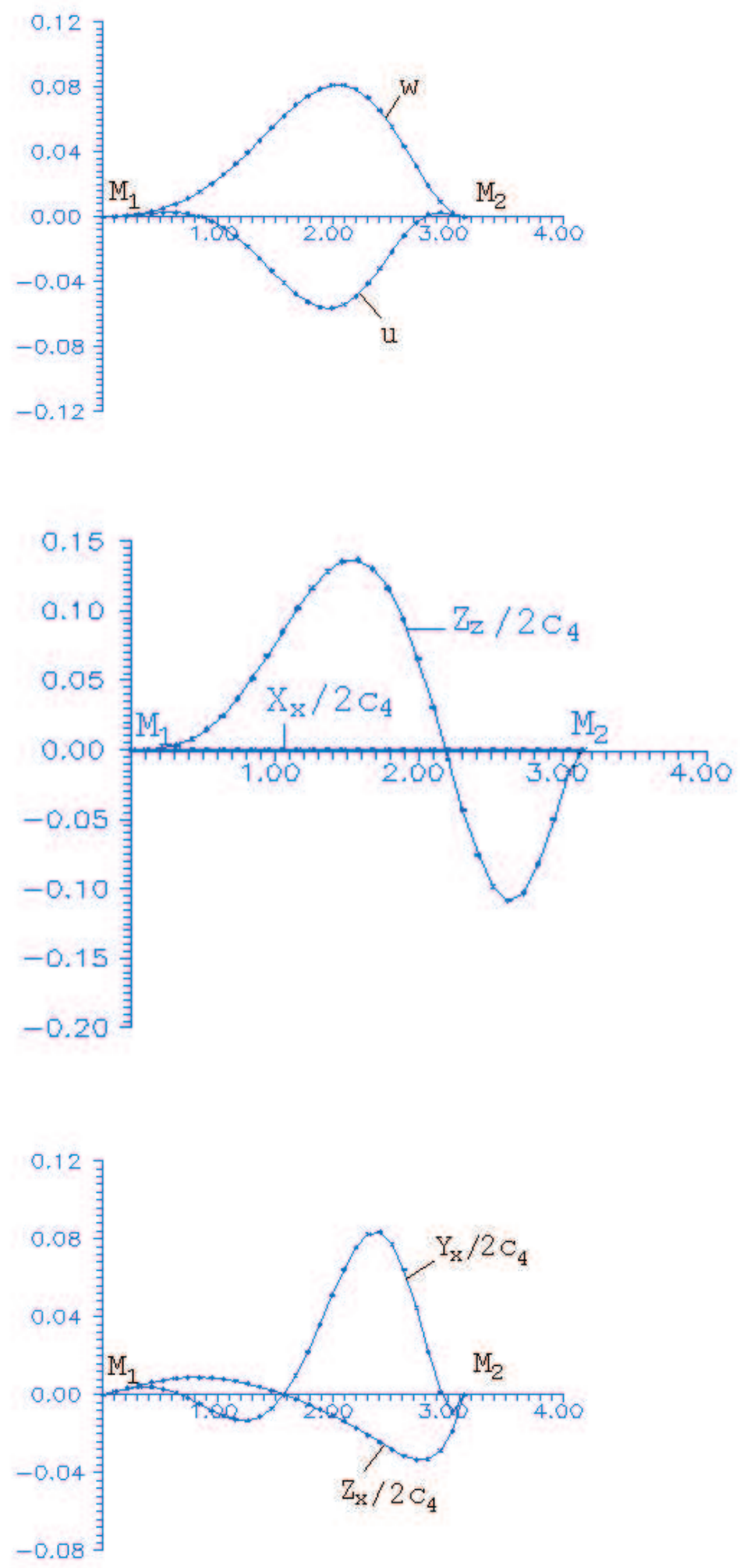

Fig. 1 


\section{REFERENCES}

1. N. Khomasuridze, Thermoelastic stress of multilayer cylindrical bodies. J. Thermal Stresses 24(2001), 919-936.

2. N. Khomasuridze, Thermoelastic equilibrium of bodies in generalized cylindrical coordinates. Georgian Math. J. 5(1998), No. 6, 521-544.

3. V. NovatskiĬ, Theory of elasticity. (Translated from the Polish into Russian) Mir, Moscow, 1975.

4. V. NovatskiI, Electromagnetic effects in solids. (Translated from the Polish into Russian) Recent Publications in Foreign Science, 37. Mir, Moscow, 1986.

5. N. Khomasuridze, Thermoelastic equilibrium of a rectangular parallelepiped with nonhomogeneous symmetry and antisymmetry conditions on its faces. Georgian Math. J. 7(2000), No 4, 701-722.

(Received 28.05.2003)

Author's address:

I. Vekua Institute of Applied Mathematics

I. Javakhishvili Tbilisi State University

2, University St., Tbilisi 0143

Georgia

E-mail: nurikhomasuridze@hotmail.com 